\title{
CdSe-ZnS Core-Shell Quantum Dots: Surface Plasmons Effect and Optical Spectra
}

\author{
Hassan H. Mohammed ${ }^{1}$ \\ ${ }^{1}$ Department of Physics, College of Science, University of Basrah, Basrah, Iraq \\ Correspondence: Hassan H. Mohammed, Department of Physics, College of Science, University of Basrah, \\ Basrah, Iraq. E-mail: hassan.mohammed@uobasrah.edu.iq
}

Received: August 5, 2013 Accepted: September 26, 2013 Online Published: October 11, 2013

doi:10.5539/apr.v5n6p15 URL: http://dx.doi.org/10.5539/apr.v5n6p15

\begin{abstract}
Absorption and photoluminescence (PL) spectra of a colloidal CdSe-ZnS core-shell quantum dots (QDs) were measured within the strong confinement regime. The QDs were casted on half-coated quartz substrates with 50 $\mathrm{nm}$ of gold and prepared for studying the surface plasmons effect. The samples were optically arranged and pumped by different wavelengths, and the PL spectra were detected. Excitation with a wavelength of $400 \mathrm{~nm}$ reveals a structure fluorescence spectrum which consists of five distinct bands. These bands are more intense and resolved than the corresponding traditional weak absorption bands. They were detected for the first time and assigned according to the theoretical predictions of excitons in a spherical potential with Coulomb interactions and valence bands mixing. A scheme based on multiple exciton generation (MEG) for the appearance of the fluorescence bands was proposed. This scheme was confirmed by the recent theoretical prediction using the state-of-the art time domain $a b$ initio density functional theory and the atomistic pseudopotential calculations. The detected surface plasmons effect in QDs enhances the intensity of the fluorescence bands. This surface plasmons effect facilitated the appearance of these mentioned bands in more intensive features than the corresponding traditional weak absorption bands. Furthermore, the reduction in the measured lifetime of the first excited electronic state from $20 \mathrm{~ns}$ for the QDs deposited directly on the quartz substrate to $7 \mathrm{~ns}$ for the QDs casted on the gold film, gives a further evidence of the surface plasmons effect in the (PL) of the CdSe-ZnS QDs.
\end{abstract}

Keywords: CdSe-ZnS QDs, surface plasmons, optical spectra

\section{Introduction}

Among many of the new inorganic semiconductor crystals that reduced to a nanometer size, CdSe quantum dots represent the prototype and most interesting material. They have therefore attracted considerable attention from the viewpoints of fundamental physics and functional device applications. ZnS-capped CdSe QDs properties have generated a great deal of interest due to the scientific aspects that are involved in these studies. Examples of interesting and significant physics include: fluorescent biological imaging probes (Mukherjee \& Ghost, 2012), solar cell devices (Kashyout et al., 2012), white light emitting diodes (Nizamoglu \& Demir, 2009), nano lasers (Yan et al., 2012), and optical fiber sensors (Jorge et al., 2009). In all these technological applications, the unique optical properties of QDs are size dependent. In addition, quantum dots are an excellent testing ground for the applicability of various theoretical models. For improving the optical properties of QDs through increasing the PL intensity, surface plasmons effect is the optimum choice. This work focuses on the surface plasmons coupled emission of CdSe-ZnS core-shell for the purpose of increasing the emission intensity and enhancing the bands that are unobservable by the traditional detection technique.

\section{Experimental Details}

A core-shell CdSe-ZnS based QDs in a toluene solution of $(50 \mathrm{mg} / \mathrm{ml})$ were purchased from Evident technologies. The QD toluene solutions were diluted five times and drop casted on half coated substrates with 50 $\mathrm{nm}$ gold by thermal evaporation. After the solutions evaporated, a thick layer of the QD nanocrystals remained on the substrates. Time-resolved fluorescence decay data were collected using the picosecond time-correlated single photon counting (TCSPC) technique (instrumental resolution function IRF $=23 \mathrm{ps}$ ). The excitation source is a picosecond Ti: sapphire laser (MIRA) from Coherent Inc. (vertical polarization, wavelength range of $720-1000 \mathrm{~nm}$, and $76 \mathrm{MHz}$ repetition rate) coupled to a second harmonic generator (360-500 nm). Emission 
collected at the polarization magic angle is detected through a double subtractive monochromator by a microchannel plate (MCPT) Hamamatsu model R3809U-50. Signal acquisition was performed using a TCSPC module (Becker \& Hickl, model SPC-630). Deconvolution of the fluorescence decays was performed using the Globals WE software package (Gratton \& Beechem, 2004).

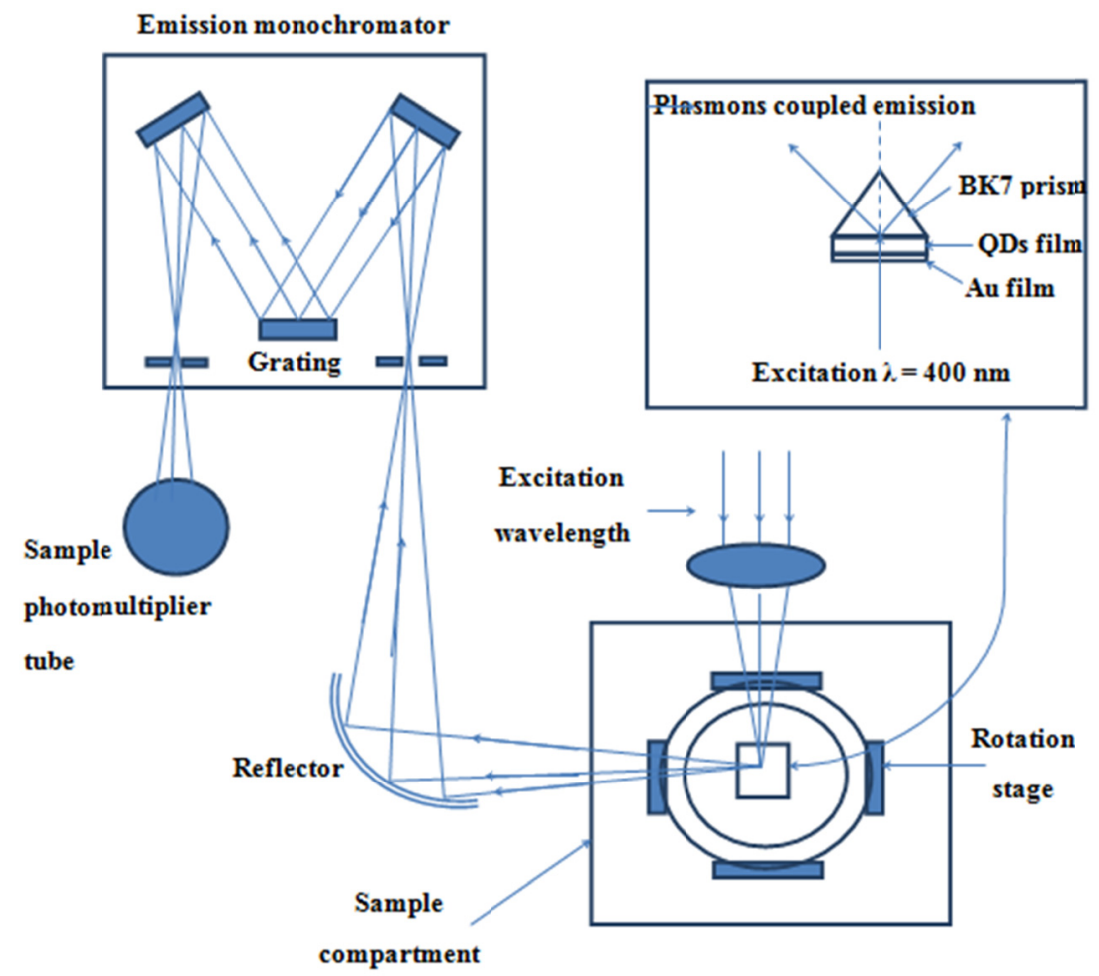

Figure 1. The experimental set-up

Absorption spectra were measured using a Varian Labomed computer control spectrophotometer. For surface plasmons resonance emission, the technique explained in Gryczynski et al. (2005) was used. The half coated slides were attached to a BK7 glass prism. This combined sample was placed on a rotary stage as shown in Figure 1 which allows excitation and observation at any desired angle relative to the vertical axis. The fluorescence spectra were detected by the Varian spectrophotometer integrated with Cary-Eclipse software for the data acquisition. XRD measurements were employed for the QDs diameter estimation where the XPERT-PRO diffractometer system which was used with Cu-KAlpha $(\lambda=1.54060 \AA)$ as an excitation source.

\section{Results and Discussion}

XRD experiments were used to characterize the crystallinity and provide a good means of measuring the average size of the nanocrystals. XRD results shown in Figure 2 which have peaks due to diffraction corresponding to (111), (220), and (311) reflections. These peaks are consistent with the zinc-blend cubic structure for CdSe (Dipesh, 2012). The XRD peaks can be used to calculate the average size of the CdSe-ZnS QDs using the well known Debye-Scherrer relationship

$$
D=\frac{0.9 \lambda}{\beta \cos \theta}
$$

Where $\mathrm{D}$ is the average crystal size, $\lambda$ is the wavelength of the $\mathrm{X}$-ray source, $\beta$ is the full width of half the maximum (FWHM) value of an XRD peak in radians, and $\theta$ is the diffracted angle. Using the values of $\beta$ and $\theta$ from the analysed XRD computer data of the Figure 2 and employing the relationship (1), the average diameter of the QDs was found to be $4 \mathrm{~nm}$. 


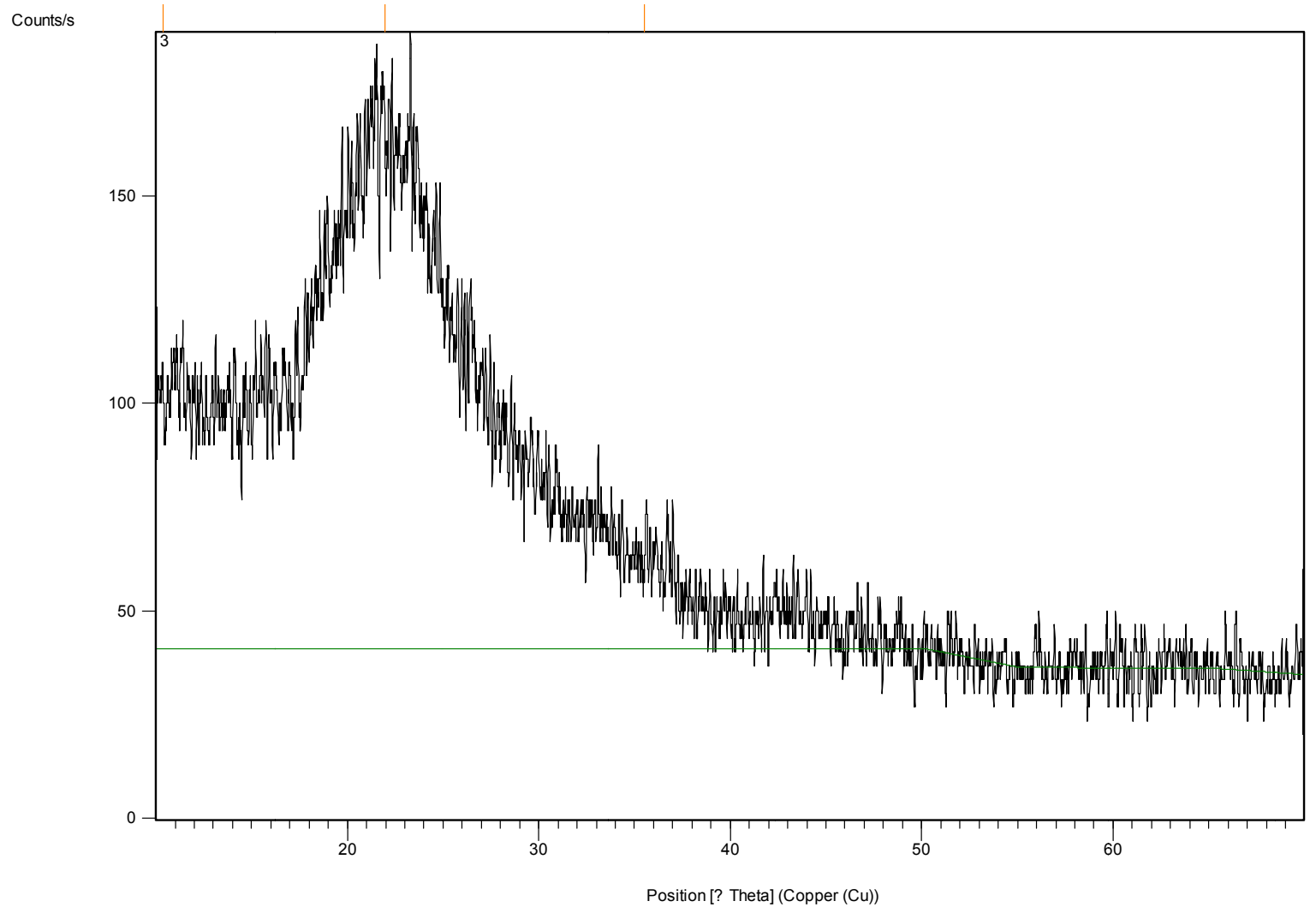

Figure 2. XRD Spectrum of CdSe-ZnS QDs

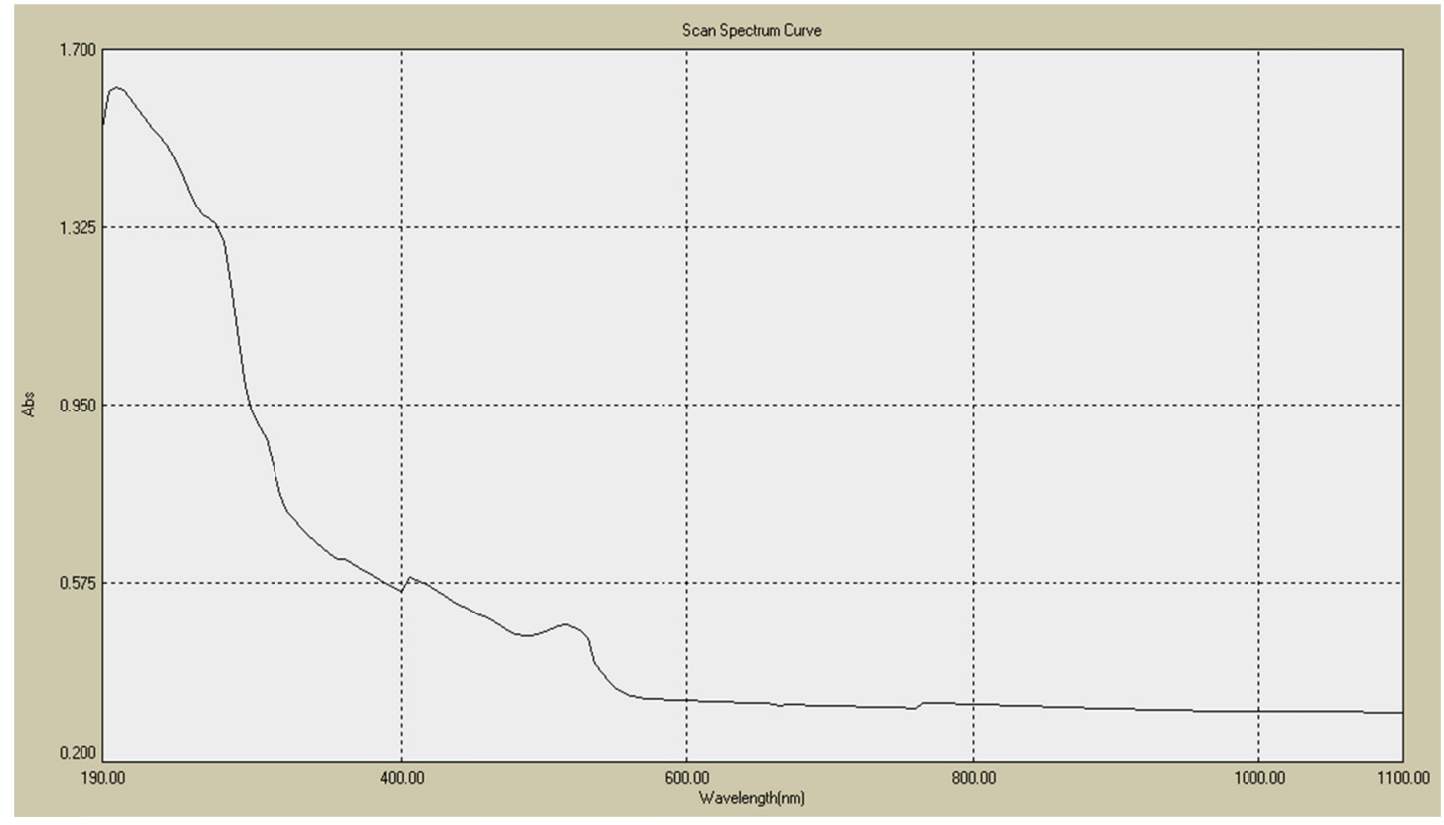

Figure 3. Absorption spectrum of CdSe-ZnS QDs

The average sizes of the QDs were also estimated by applying an empirical relationship (Nguyen, 2010)

$$
\begin{gathered}
D=\left(1.6122 \times 10^{-9}\right) \lambda^{4}-\left(2.6575 \times 10^{-6}\right) \lambda^{3}+\left(1.6242 \times 10^{-3}\right) \lambda^{2}-(0.4277) \lambda \\
+41.57
\end{gathered}
$$


Where $\lambda$ is the wavelength of the first excitonic absorption peak. The absorption spectrum of the sample is shown in Figure 3. Five traditional weak absorption bands were appeared in this spectrum. The first absorption peak is more resolved than the others and situated at $526 \mathrm{~nm}$. Applying the relationship (2), the estimated value of $\mathrm{D}=4.049 \mathrm{~nm}$. Comparing the $\mathrm{D}$ value calculated from the XRD measurements with that estimated from the optical absorption spectra, the average size of the QDs was $4.02 \mathrm{~nm}$. This QDs size covers the strong confinement regime, where the confinement energies of the electron and hole are much larger than the Coulomb interactions. The first excitonic energy as a function of the radius of the nanocrystal can be expressed as Woggon (1997)

$$
E=E_{\mathrm{g}}+\frac{\hbar^{2} \pi^{2}}{2 \mathrm{R}^{2}}\left(\frac{1}{m_{e}^{*}}+\frac{1}{m_{h}^{*}}\right)-\frac{1}{\mathrm{R}}\left(\frac{1.8 e^{2}}{4 \pi \varepsilon_{0} \varepsilon_{r}}\right)
$$

Where $\mathrm{m}_{\mathrm{e}}{ }^{*}$ and $\mathrm{m}_{\mathrm{h}}{ }^{*}$ are the effective masses of the electron and hole, $\mathrm{R}$ is the radius of the QDs. For the bulk $\mathrm{CdSe}$, the energy gap $\mathrm{E}_{\mathrm{g}}$ is $1.7 \mathrm{eV}, \mathrm{m}_{\mathrm{e}}{ }^{*} / \mathrm{m}_{0}$ is $0.13, \mathrm{~m}_{\mathrm{h}}{ }^{*} / \mathrm{m}_{0}$ is 0.45 and the dielectric constant of the surrounding medium $\varepsilon_{\mathrm{r}}$ is 10.6 , according to Biswas and Ghosel (2011), $\mathrm{h}=\mathrm{h} / 2 \pi, \mathrm{h}$ is Plank's constant, $\varepsilon_{0}$ is the permittivity of free space and e is the electron mass. Then the photon energy in $\mathrm{eV}$ can be estimated from the radius in nm. The estimated value of the first excitonic energy is $1.866 \mathrm{eV}$, while the measured energy by the absorption peak is $2.30 \mathrm{eV}$. The calculated value is less than the measured one by $0.44 \mathrm{eV}$. This discrepancy is due to the following reasons: (i) the approximations involved in the well-known theoretical model using the effective mass approximation with e-h pair is confined at the center of an infinite spherical potential well and (ii) the neglect of the QDs levels populations according to the (MEG) via multiple pathways as that shown in the proposed mechanism illustrated in the Scheme 1. The positions of the other four weak excitonic peaks appeared in Figure 3 along with the assignment associated with each peak is given in the Table 1 . The first four peaks with their assignments are in agreement with that observed by Klimov (2007) for the CdSe nanocrystal of $4.1 \mathrm{~nm}$ in diameter. The fifth weak band is observed in this work and assigned to the transition $1 \mathrm{~S}_{1 / 2}(\mathrm{~h})-1 \mathrm{~S}(\mathrm{e})$. This assignment is based on the order of increasing energy of the successive CdSe bands according to Norris and Bawendi (1996). This theoretical prediction demonstrates the importance of the valence band structure in the description of the excitonic energy levels in the valence band. This transition is situated directly after the previous $3 \mathrm{~S}_{1 / 2}-1 \mathrm{~S}(\mathrm{e})$ transition and is therefore the more pronounced among the others.

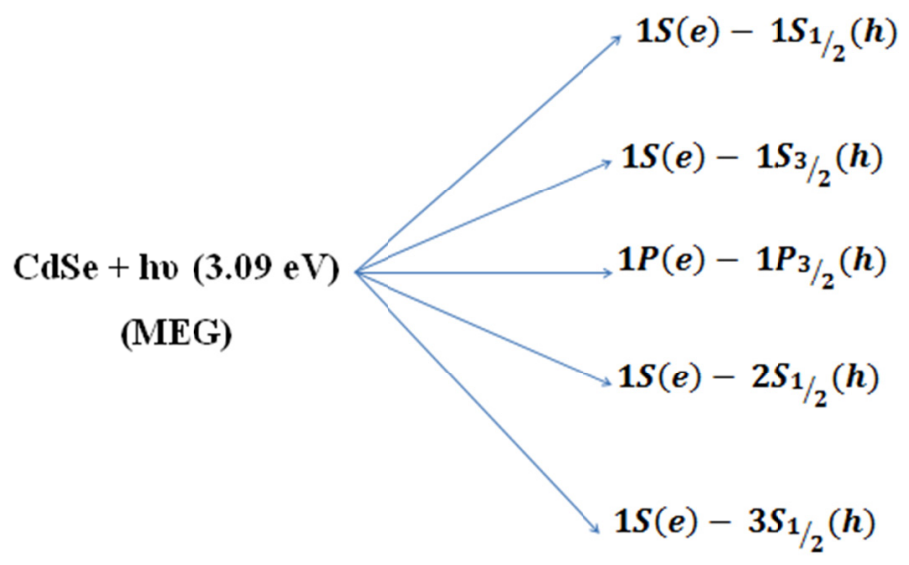

Scheme 1. The proposed mechanism of the levels population of the CdSe-ZnS QDs

Table 1. Peak positions of five assigned bands of CdSe-ZnS core-shell quantum dots

\begin{tabular}{cccc}
\hline \multirow{2}{*}{ Transitions } & \multicolumn{3}{c}{ CdSe-ZnS QDs Sample } \\
\cline { 2 - 4 } & Abs. peak $(\mathrm{eV})$ & Emission peak $(\mathrm{eV})$ & Stokes shift $(\mathrm{eV})$ \\
\hline $1 S_{3 / 2}(h)-1 S(e)$ & 2.308 & 2.145 & 0.163 \\
$2 S_{3 / 2}(h)-1 S(e)$ & 2.984 & 2.208 & 0.776 \\
$1 P_{3 / 2}(h)-1 P(e)$ & 3.373 & 2.291 & 1.082 \\
$3 S_{1 / 2}(h)-1 S(e)$ & 4.048 & 2.530 & 1.518 \\
$1 S_{1 / 2}(h)-1 S(e)$ & 4.588 & 2.669 & 1.919 \\
\hline
\end{tabular}




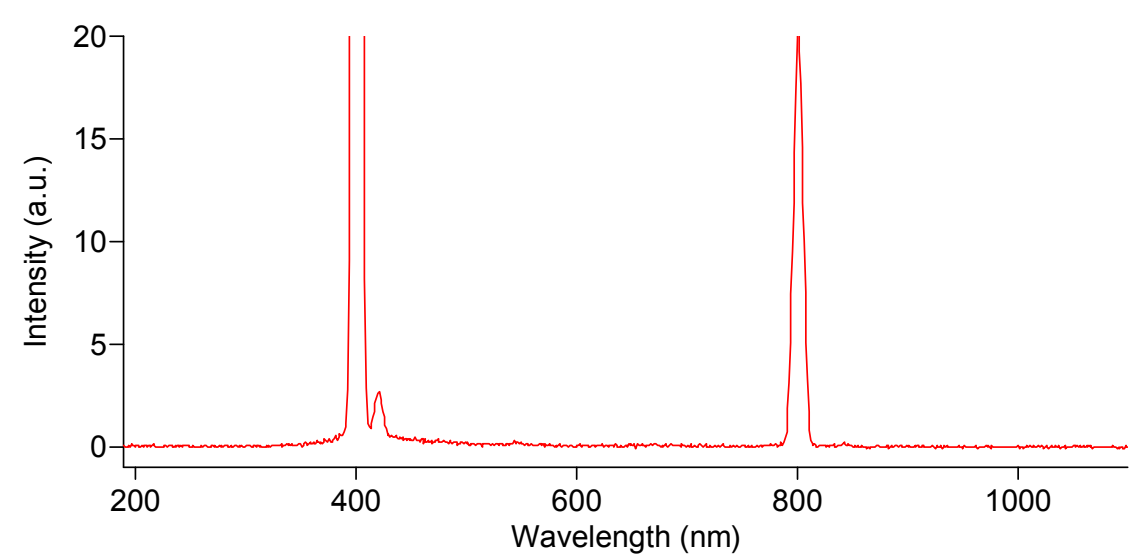

Figure 4. The spectrum of the excitation source for fluorescence measurements

In order to distinguish the fluorescence bands from the excitation source bands, the excitation source emission bands are presented in Figure 4. The PL spectrum is shown in Figure 5 for two cases: QDs deposited on gold and on quartz respectively. The PL spectrum is more intense for QDs deposited on gold than for QDs deposited on quartz due to surface plasmons effect.These spectra were repeated many times and found reproducible. The first excitonic peak situated at $660 \mathrm{~nm}$ is the traditional peak usually observed for such QDs. The other peaks were never detected. By investigating these features, the most interesting point to be considered is that the spacing between the level $\mathrm{S}(\mathrm{e})$ and $\mathrm{P}(\mathrm{e})$ is large. The density of states feature is shown to be in coincidence with the recent time domain density functional theory of CdSe-ZnS QDs developed by Kilina, Killin, and Prezhdo (2009). According to this approach the CdSe-ZnS density of states (DOS) shows asymmetry across the band gap, with the hole states having a higher density than the electron states and a larger spacing between $1 \mathrm{~S}(\mathrm{e})$ and $1 \mathrm{P}(\mathrm{e})$ levels. The distinct and well separated peaks observed for the first time in fluorescence shown in Figure 5 is in the contrary to effective mass description, which predicts that electronic energy levels are highly degenerate. Figure 6 shows the energy levels involved in the absorption and fluorescence of $\mathrm{CdSe}-\mathrm{ZnS}$ core-shell quantum dots. The atomistic simulation of Kilina et al. (2009) shows that the underlying atomic structure, surface effects, core-shell interaction, as well as spin-orbit interaction, break the degeneracy and create a multilevel electronic structure. These simulation results are in a good agreement with the present fluorescence experimental data. Another interesting feature, which supports the observed structure spectrum in fluorescence, is that simulation results reported by Puzder et al. (2004). This simulation proves that the DOS for unrelaxed CdSe QDs is identical to the corresponding relaxed QDs. This theoretical prediction gives a strong evidence for the observation of the fluorescence structure as approximate mirror image to the absorption features. The discrete bands observed in fluorescence can be explained by the multiple exciton generation (MEG). Excitons in CdSe-ZnS QDs relax via multiple pathways according to the Scheme 1.

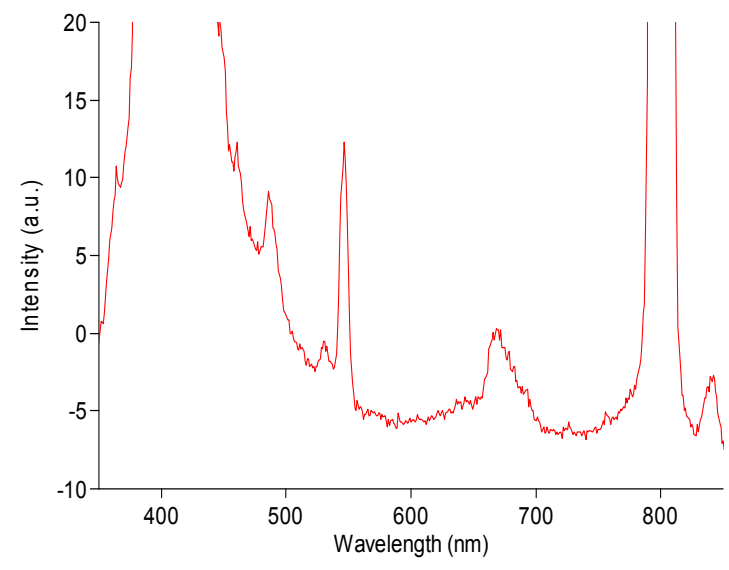

a- QDs on gold

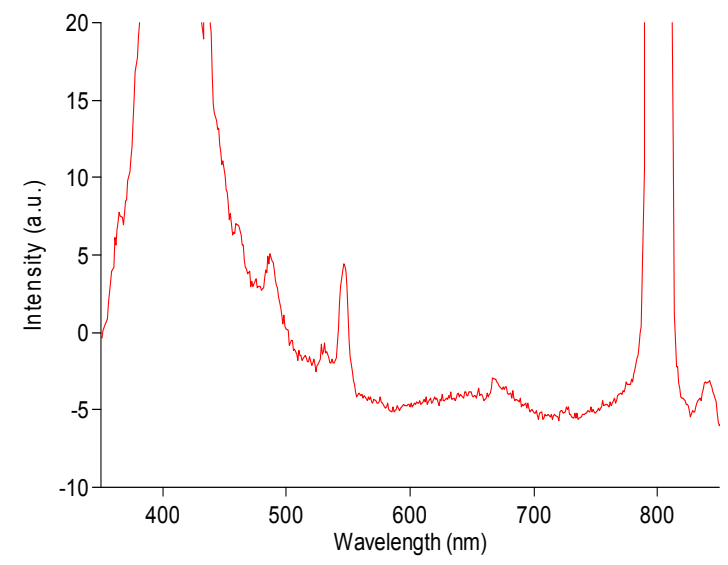

b- QDs on quartz

Figure 5. The PL spectrum of a-CdSe-ZnS QDs on gold film b-on quartz substrate 
The simulation results of Prezhdo (2008) indicate that MEG can be created directly upon absorption of a single photon. Furthermore MEG in CdSe QDs can appear at energies of $3 \mathrm{eV}$ and above Prezhdo (2008). The simulation results support the proposed mechanisms for the levels populations and subsequent relaxations.

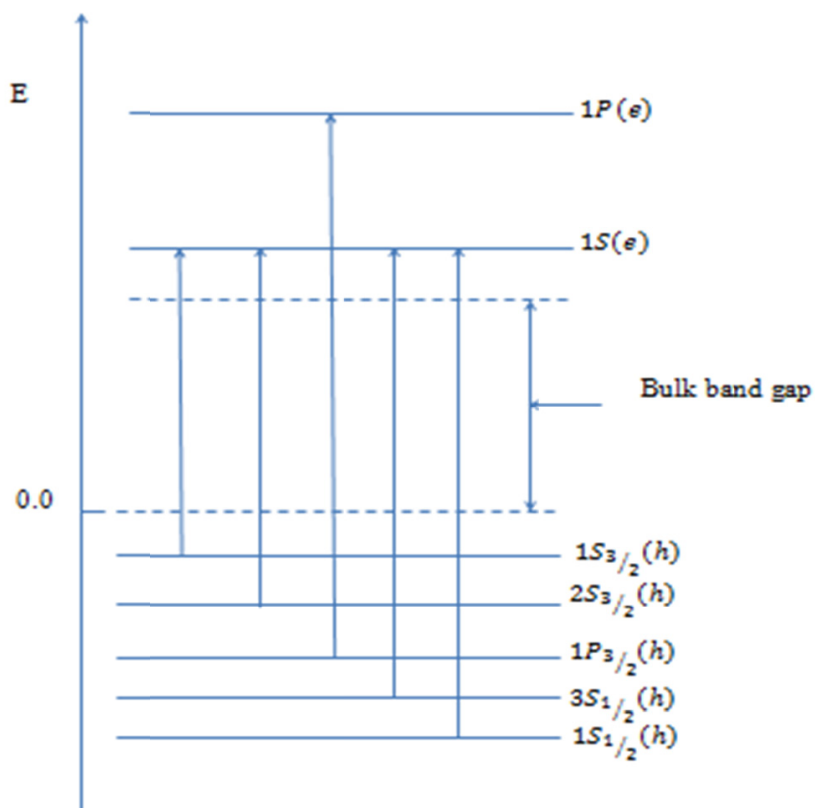

Figure 6. Energy levels involved in the absorption and fluorescence of $\mathrm{CdSe}-\mathrm{ZnS}$ core-shell quantum dots

A very large Stokes shift was observed in the luminescence with respect to absorption for the excitation of 3.09 $\mathrm{eV}$, which is far from the band edge (nonresonant excitation). The results show that for the lowest excitonic state, the Stokes shift is $0.163 \mathrm{eV}$, which is greater by about a factor of two. This is in a good agreement with the experimental and theoretical predictions of Efros et al. (1996). Stokes shift ratios for the higher excitonic states given in Table 1 are: $0.926,0.842,0.661$, and 0.770 which are less pronounced than the first excitonic states. These results demonstrate that, while the first absorption peak generally follows the crystal size distribution, where the position of the luminescence line for nonresonant excitation is determined by the largest crystal within this distribution. This causes a very large Stokes shift for the lowest excitonic states because the energy of the band edge transitions in larger crystal is less than that in smaller ones.

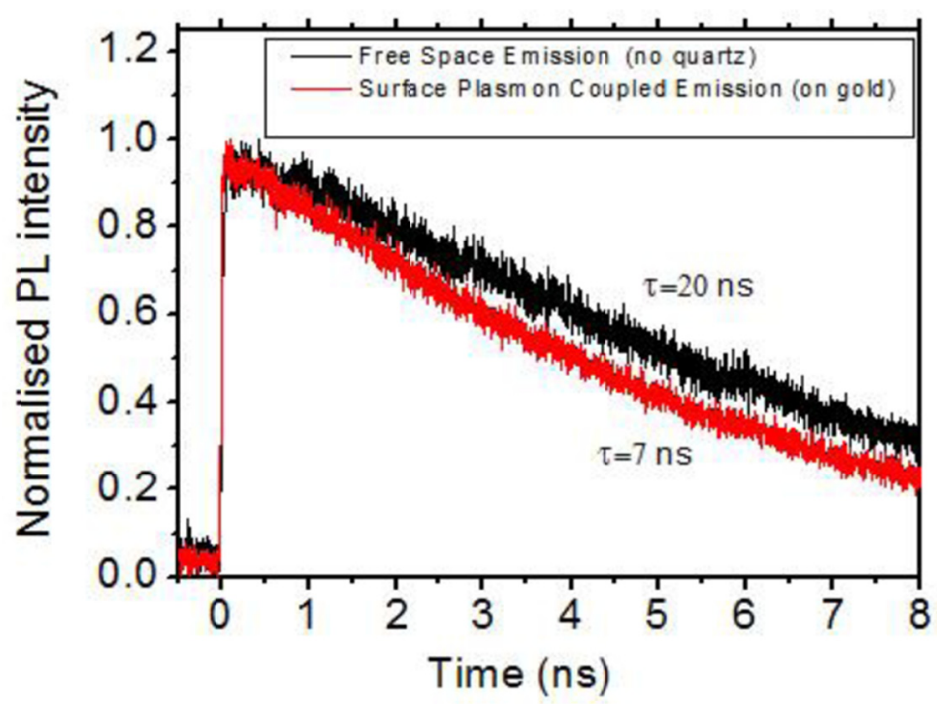

Figure 7. The lifetimes of the first excitonic state of CdSe-ZnS QDs: on gold film and on quartz substrate 
Figure 7 shows that the QDs on the gold showing shorter lifetime 7 ns compared with that on the quartz 20 ns. Comparing with our measurement of the steady state PL emission (Figure 5) which indicates an enhancement for the sample on gold. This suggests an enhancement in the radiative decay due to surface plasmons effect. It has been shown that surface magneto-optical interactions and dipole-dipole interactions between nanoparticles can be enhanced using surface plasmons Avrutsky (2004). The spontaneous emission lifetime can be calculated from the homogeneous radiative decay rate via Leistikow et al. (2009);

$$
\tau_{21}=\frac{6 m_{e} \varepsilon_{0} \pi c^{3}}{\mathrm{e}^{2} \mathrm{n} \omega^{2} \mathrm{f}_{\mathrm{osc}}}
$$

Where $f_{\text {osc }}$ is the emission oscillator strength, $m_{e}$ is the electron mass, $\varepsilon_{0}$ is the vacuum permittivity, $c$ is the speed of light, $\omega$ is the angular frequency of the first excitonic transition and $n$ is the refractive index of the surrounding medium. The value of $\mathrm{n}$ at the emission wavelength is 2.25 (Wu et al., 2008) and the experimental value of $\mathrm{f}_{\mathrm{osc}}$ is 0.45 (Leistikow et al., 2009). The calculated value of the radiative spontaneous lifetime of the QDs by using Equation 4 is $19.47 \mathrm{~ns}$, which is close to the experimental value. This lifetime value is shown to be reduced by a factor of 3 compared to QDs deposited on the gold film due to enhancement in the emission intensity. Such an effect gives a further evidence for the surface plasmons effect. The present experimental results will open the way for further investigations in nanoscale electronic structures.

\section{Conclusions}

This work shows that the CdSe-ZnS QDs response to external perturbations such as surface plasmons effect depends strongly on the excitation wavelength along with the optical arrangement for the fluorescence collection. Both factors are shown manifest themselves and intimately intertwined in a detection of the fluorescence spectra and subsequent energy levels determination. The relaxed and unrelaxed features of the QDs are shown in a full agreement with the recent theoretical predictions using the time domain density functional theory and the atomistic pseudopotential calculations. The present experimental results will be very useful for applied scientific and experimental research programs.

\section{References}

Avrutsky, I. (2004). Surface plasmons at nanoscale relief gratings between a metal and a dielectric medium with optical gain. Physical Review B, 70, 155416, 1-6. http://dx.doi.org/10.1103/PhysRevB.70.155416

Biswas, A., \& Ghosel, A. (2011). Hole transport characteristics of CdSe single quantum wells at low temperatures. Journal of Electron Devices, 10, 444-447. Retrieved from http://www.jeldev.org/10Biswas2.pdf

Dipesh, N. (2012). Structural and optical investigation of CdSe quantum dots. Kathmandu University Journal of Science, Engineering and Technology, 8(II), 83-88. Retrieved from www.ku.edu.np/kuset/vol8_no2/12_dipesh\%20neupane.pdf

Efros, Al. L., Rosen, M., Kuno, M., Nirmal, M., Norris, D. J., \& Bawendi, M. G. (1996). Band-Edge exciton in quantum dots of semiconductors with a degenerate valence band: Dark, and bright exciton states. Physical Review B, 54, 4843-4856. http://dx.doi.org/10.1103/PhysRevB.54.4843

Gratton, E., \& Beechem, J. (2004). Globals WE (software), Laboratory for fluorescence dynamics, University of California, Irvine, CA.

Gryczynski, I., Malicka, J., Jiang, W., Fischer, H., Chan, W. C. W., Gryczynski, Z., ... Lakowicz, J. R. (2005). Surface plasmons coupled emission of quantum dots. Journal of Physical Chemistry B, 109(3), 1088-1093. http://dx.doi.org/10.1021/jp046173i

Jorge, P., Martins, M. A., Trindade, T., Santos, J. L., \& Farahi, F. (2007). Optical fiber sensors using quantum dots. Sensors, 7(12), 3489-3534. http://dx.doi.org/10.3390/s7123489

Kashyout, A. B., Soliman, H. M. A., Fathy, M., Gomaa, E. A., \& Zidan, A. A. (2012). CdSe quantum dots for solar cells devices. International Journal of Photoenergy, 2012, 1-7. http://dx.doi.org/10.1155/2012/952610

Kilina, S. V., Killin, D. S., \& Prezhdo, O. V. (2009). Breaking the phonon bottlneck in PbSe and CdSe quantum dots: Time-Domain density functional theory of charge carrier relaxation. ACS Nano, 3(1), 93-99. http://dx.doi.org/10.1021/nn800674n

Klimov, V. I. (2007). Spectral and dynamical properties of multiexcitons in semiconductor nanocrystals. Annual Review of Physical Chemistry, 58, 635-673. http//dx.doi.org/10.1146/annurev.physchem.58.032806.104537 
Leistikow, M. D., Johansen, J., Kettelarij, A. J., Lodahl, P., \& Vos, W. L. (2009). Size-Dependent oscillator strength and quantum efficiency of CdSe quantum dots controlled via the local density of states. Physical Review B, 79, 045301, 1-9. http://dx.doi.org/10.1103/PhysRevB.79.045301

Mukherjee, A., \& Ghost, S. (2012). Optimum excitation photon energy for CdSe-ZnS core-shell quantum dots based luminescence imaging. Journal of Physics D: Applied Physics, 45(19), 1-5. http://dx.doi.org/10.1088/0022-3727/45/19/195103

Nizamoglu, S., \& Demir, H. V. (2009). Excitation color conversion of CdSe/ZnS core/shell quantum dots solids for white light emitting diodes. Journal of Applied Physics, 105, 083112, 1-5. http://dx.doi.org/10.1063/1.3109151

Nguyen, H. Q. (2010). Synthesis and optical properties of CdSe nanocrystal and CdSe/ZnS core/shell nanostructure in non-coordinating solvents. Advances in Natural Sciences: Nanoscience and Nanotechnology, 1(2), 1-4. http://dx.doi.org/10.1088/2043-6254/1/2/025004

Norris, D. J., \& Bawendi, M. G. (1996). Measurement and assignment of the size-dependent optical spectrum in CdSe quantum dots. Physical Review B, 53(24), 16338-16346. http://dx.doi.org/10.1103/PhysRevB.53.16338

Puzder, A., Williamson, A., Gygi, F., \& Galli, G. (2004). Simulations of CdSe quantum dots. UCRL-CONF-203007. Retrieved from http://e-reports.ext.llnl.gov./306048

Prezhdo, O. V. (2008). Multiple excitons and the electron-phonon bottleneck in semiconductor quantum dots: $a b$ initio perspective. Chemical Physics Letters, 460(1-3), 1-9. http://dx.doi.org/10.1016/j.cplett.2008.03.099

Woggon, U. (1997). Optical properties of semiconductor quantum dots. Springer Verlag GmbH.

Wu, X., Lai, F., Lin, L., Lv, J., Zhuang, B., Yan, Q., \& Huang, Z. (2008). Optical inhomogeneity of ZnS films deposited by thermal evaporation. Applied Surface Science, 254(20), 6455-6460. http://dx.doi.org/10.1016/j.apsusc.2008.04.023

Yan, J. H., Wang, C. G., Zhang, H., \& Cheng, C. (2012). Evaluation of emission cross section of CdSe quantum dots for laser applications. Laser Physics Letters, 9(7), 529-535. http://dx.doi.org/10.7452/lapl.201210037

\section{Copyrights}

Copyright for this article is retained by the author(s), with first publication rights granted to the journal.

This is an open-access article distributed under the terms and conditions of the Creative Commons Attribution license (http://creativecommons.org/licenses/by/3.0/). 